\title{
Symposium
}

\section{Carcinoma pancreas}

\author{
Col K.J. Singh ${ }^{a, *}$, Col Ashwin Galagali ${ }^{a}$, Maj G. Menon ${ }^{b}$ \\ ${ }^{a}$ Associate Professor, Department of Surgery, AFMC, Pune 40, India \\ ${ }^{\mathrm{b}}$ Clinical Tutor, Department of Surgery, AFMC, Pune 40, India
}

\section{Introduction}

Carcinoma Pancreas ranks fourth among cancer-related deaths in the United States. Carcinoma Pancreas is more common in elderly persons than in younger persons, and less than $20 \%$ of patients present with localized, potentially curable tumors. The overall 5-year survival rate among patients with pancreatic cancer is $<5 \% .{ }^{1}$ In recent years, there have been important advances in the understanding of the molecular biology of pancreatic cancer as well as in diagnosis, staging, and treatment in patients with early-stage tumors. Minimal progress has been made, however, in prevention, early diagnosis, and treatment in patients with advanced disease.

\section{Etiology}

The causes of Carcinoma Pancreas remain unknown. Approximately $5-10 \%$ of patients with pancreatic cancer have a family history of the disease. ${ }^{2}$ In some patients, pancreatic cancer develops as part of a well-defined cancer-predisposing syndrome for which germ-line genetic alterations are known. The risk of pancreatic cancer is 57 times as high in families with four or more affected members as in families with no affected members. A subgroup of such high-risk kindred carry germ-line mutations of DNA repair genes such as BRCA2 and the partner and localizer of BRCA2 (PALB2). ${ }^{3,4}$

Several environmental factors have been implicated, but evidence of a causative role exists only for tobacco use. Smokers have a $2.5-3.6 \%$ increased risk and this increases with greater tobacco use and longer exposure to smoke. Limited data with increased incidence is available on the possible roles of moderate intake of alcohol, coffee, use of aspirin, history of diabetes or chronic pancreatitis, chronic cirrhosis, a high-fat, high-cholesterol diet, and previous cholecystectomy. ${ }^{5-7}$ More

recently, an increased risk has been observed among patients with blood type $\mathrm{A}, \mathrm{B}$, or $\mathrm{AB}$ as compared with blood type $\mathrm{O}^{8}$

\section{The biology of pancreatic cancer}

A recent comprehensive genetic analysis of 24 pancreatic cancers showed that the genetic basis of pancreatic cancer is extremely complex and heterogeneous. In that study, an average of 63 genetic abnormalities per tumor, mainly point mutations, were classified as likely to be relevant. Data suggest that pancreatic cancer results from the successive accumulation of gene mutations. The cancer originates in the ductal epithelium and evolves from pre-malignant lesions to fully invasive cancer. The lesion called pancreatic intraepithelial neoplasia is the best-characterized histologic precursor of pancreatic cancer. ${ }^{9}$ The progression from minimally dysplastic epithelium (pancreatic intraepithelial neoplasia grades $1 \mathrm{~A}$ and 1B) to more severe dysplasia (pancreatic intraepithelial neoplasia grades 2 and 3) and finally to invasive carcinoma is paralleled by the successive accumulation of mutations that include activation of the KRAS2 oncogene, inactivation of the tumor-suppressor gene CDKN2A which encodes the inhibitor of cyclin-dependent kinase 4 (INK4a), and last, inactivation of the tumor-suppressor genes tp53 and deleted in pancreatic cancer 4 DPC4, also known as the SMAD family member 4 gene (SMAD4). Other pre-malignant lesions of the pancreas, which are less well characterized, include intrapancreatic mucinous neoplasia and mucinous cystic neoplasia. The role of angiogenesis in pancreatic cancer remains controversial.

\section{Clinical features}

At presentation, most patients have systemic manifestations of the disease such as asthenia, anorexia, and weight loss.

\footnotetext{
* Corresponding author.

E-mail address: drkjsingh@rediffmail.com (K.J. Singh). 0377-1237/\$ - see front matter @ 2012, Armed Forces Medical Services (AFMS). All rights reserved. doi:10.1016/j.mjafi.2012.04.013
} 
Other manifestations include deep and superficial venous thrombosis, panniculitis, features of obstructive jaundice, gastric outlet obstruction, increased abdominal girth, and depression. Obstruction of the pancreatic duct may lead to pancreatitis. Patients with pancreatic cancer often have dysglycemia. Indeed, pancreatic cancer should be considered in the differential diagnoses of acute pancreatitis and newly diagnosed diabetes.

Physical examination may reveal jaundice, temporal wasting, peripheral lymphadenopathy, hepatomegaly, and ascites.

\section{Evaluation}

Evaluation of a patient in whom pancreatic cancer is suspected focuses on diagnosis and staging of the disease, assessment of resectability, and palliation of symptoms. Routine blood tests may reveal abnormalities in liver-function tests, hyperglycemia, and anemia. ${ }^{10}$

Ultrasound as an initial modality followed by multiphase, multidetector helical contrast enhanced computed tomography (MDCECT) is the imaging procedure of choice. This allows visualization of the tumor in relation to the superior mesenteric artery, celiac axis, superior mesenteric vein and portal vein. It also allows assessment of metastasis and ascites. It is sufficient to confirm a suspected pancreatic mass and to frame an initial management plan. Overall, MDCECT predicts surgical resectability with $80-90 \%$ accuracy. ${ }^{11}$ Positron-emission tomography can be useful if the CT findings are equivocal.

Some patients require additional diagnostic studies. Endoscopic ultrasonography (EUS) is useful in patients in whom pancreatic cancer is suspected although there is no visible mass identifiable on CT or there is a head mass with possibility of chronic pancreatitis. It is the preferred method of obtaining tissue for diagnostic purposes. Although a tissue diagnosis is not needed in patients who are scheduled for surgery, it is required before the initiation of treatment with chemo or radiation therapy. Endoscopic retrograde cholangiopancreatography (ERCP) can be used to guide ductal brushing and lavage, which provides tissue for diagnosis. It is useful in patients with jaundice in whom an endoscopic stent is required to relieve obstruction. ${ }^{12}$ In patients who have large tumors, especially in the body and tail of the pancreas, as well other indications of advanced disease such as weight loss, an elevated level of carbohydrate antigen 19-9 (CA 19-9), ascites, or equivocal CT findings, a staging laparoscopy can accurately determine metastatic and vascular involvement. ${ }^{13}$
CA 19-9 is the only biomarker with demonstrated clinical usefulness and is useful for therapeutic monitoring and early detection of recurrent disease after treatment in patients with known pancreatic cancer. ${ }^{14-16}$ However, CA 19-9 is not a specific biomarker for pancreatic cancer; its level may be elevated in other conditions such as cholestasis. In addition, patients who are negative for Lewis antigen a or b (approximately $10 \%$ of patients with pancreatic cancer) are unable to synthesize CA 19-9 and have undetectable levels, even in advanced stages of the disease. Although measurement of serum CA 19-9 levels is useful in patients with known pancreatic cancer, the use of this biomarker as a screening tool has had disappointing results.

Universal primary screening for pancreatic cancer is currently not recommended, given the tools available and their performance. ${ }^{17}$

\section{Staging of pancreatic cancer}

Pancreatic cancer is staged according to the most recent edition of the American Joint Committee on Cancer tumor-nodemetastasis classification, which is based on assessment of resectability by means of helical $\mathrm{CT} .{ }^{18} \mathrm{~T} 1, \mathrm{~T} 2$, and $\mathrm{T} 3$ tumors are potentially resectable, whereas $\mathrm{T} 4$ tumors, which involve the superior mesenteric artery or celiac axis, are unresectable (Table 1). Tumors involving the superior mesenteric veins or splenic veins are classified as T3, since these veins can be resected and reconstructed, provided that they are patent.

\section{Management of early disease}

Patients with pancreatic cancer are best cared for by multidisciplinary teams that include surgeons, medical and radiation oncologists, radiologists, gastroenterologists, nutritionists, and pain specialists, among others. ${ }^{19,20}$ For patients with resectable disease, surgery remains the treatment of choice. Depending on the location of the tumor, the operative procedures may involve pancreatoduodenectomy (the Whipple procedure), distal pancreatectomy, or total pancreatectomy. A minimum of 12-15 lymph nodes should be resected, and every attempt should be made to obtain a tumor-free margin. Data from several randomized clinical trials indicate that a more extensive resection does not improve survival but increases postoperative morbidity. Recent studies show that the results of vein resection and vascular reconstruction in patients with limited involvement of the superior mesenteric vein and portal vein are similar

Table 1 - Staging of carcinoma pancreas.

\begin{tabular}{lcccl} 
Stage & Tumor grade & Nodal status & Metastasis & \multicolumn{1}{c}{ Characteristics } \\
\hline IA & T1 & N0 & M0 & Tumor limited to pancreas $<2 \mathrm{~cm}$ \\
IB & T2 & N0 & M0 & Tumor limited to pancreas $>2 \mathrm{~cm}$ \\
IIA & T3 & N0 & M0 & Tumor outside pancreas but not involving celiac axis or SMA \\
IIB & T1-3 & N1 & M0 & Regional lymph-node metastasis \\
III & T4 & N0-1 & M0 & Tumor involves celiac axis or SMA (unresectable) \\
IV & T1-4 & N0-1 & M1 & Distant metastasis \\
\hline
\end{tabular}


to the results in patients without vein involvement. Partial involvement of the SMV and/or SMA on CT angiography is associated with a resectability rate of $10-50 \%$ depending on the extent of vascular encroachment, although involvement of the SMA is generally a contraindication to resection. Additionally, metastasis to the liver, peritoneum, and extra-abdominal sites are all contraindications to resection. Tseng and colleagues described major vascular resection of the superior mesenteric or portal veins performed at the time of pancreaticoduodenectomy for pancreatic cancer. In their study, vein resection was performed in 141 patients in whom the tumor could not be separated from the vein. The resections included tangential resection with vein patch in 36 patients, segmental resection with primary anastomosis in 35 patients, and segmental resection with autologous interposition grafts in 55 patients. The authors compared all patients who underwent pancreaticoduodenectomy with vein resection to all patients who underwent standard resection. The need for vein resection had no impact on survival duration. ${ }^{21}$ These data support the use of vein resection as a therapeutic option in selected patients.

Poor prognostic factors include lymph-node metastases, a high tumor grade, a large tumor, high levels of CA 19-9, and positive margins of resection. ${ }^{22,23}$

Up to $70 \%$ of patients with pancreatic cancer present with biliary obstruction, which can be relieved by percutaneous or endoscopic stent placement. Operable lesions should not be stented preoperatively. Stenting has been shown to be an independent poor prognostic marker in terms of causing infection and postoperative morbidity and mortality. Decompression is appropriate for patients in whom surgery is delayed, such as patients who are treated with neo-adjuvant therapy before resection or who are referred to other centers for treatment. ${ }^{24}$ In addition, patients with cholangitis require decompression as well as antibiotic treatment before surgery. If the bilirubin level is very high (usually above $20 \mathrm{mg} \%$ ), some surgeons and centers would get the patients stented as a matter of policy for a period of 3-4 weeks prior to surgery.

Postoperative chemotherapy with gemcitabine or fluorouracil and leucovorin improves progression-free and overall survival. Varying combinations of the drugs are being tried. Large tumor size, high differentiation grade and involvement of lymph nodes are risk factors for recurrent disease. For positive margins, gemcitabine alone or in combination with fluorouracil-based chemoradiation can be considered the standard of care. The Radiation Therapy Oncology Group trial 97-94 showed that the combination of gemcitabine with fluorouracil administered as a continuous infusion and radiation therapy resulted in a trend toward increased overall survival, although the increase was not significant among patients with tumors in the head of the pancreas. These results are similar to those of large single institution series that incorporated radiation therapy. ${ }^{25}$ An emerging strategy in patients with resectable carcinoma pancreas is the use of neo-adjuvant treatment especially in patients with borderline resectable tumors.

\section{Outcome}

Even if the tumor is fully resected, the outcome in patients with early pancreatic cancer is disappointing. The outcome in patients in various trials showed a median survival of 20-22 months. Large tumor size, high differentiation grade, and involvement of the lymph nodes are risk factors for recurrent disease. The effect of positive resection margins, however, is more controversial. ${ }^{26}$

\section{Management of locally advanced and systemically advanced disease}

Approximately $30 \%$ of patients with pancreatic cancer are locally advanced at diagnosis while a similar number have local recurrence after treatment of early disease. The treatment is palliative with median survival ranging from 9 to 10 months. Management options range from systemic chemotherapy alone to combined forms of treatment. In addition stenting or surgical bypass for jaundice and gastric outlet obstruction may be considered.

A significant number of patients with pancreatic cancer either present with metastatic disease or metastasis develops in them mainly in the liver and peritoneal cavity. The treatment of these patients remains palliative although these patients should be allowed to participate in clinical trials.

\section{Future directions}

There is significant room for improvement in all aspects of the management of pancreatic cancer. Screening of high-risk persons by either innovative imaging methods or measurement of serum biomarkers for picking up the disease early is critical. A better understanding of tumor biology resulting in new targeted agents including monoclonal antibodies targeting cell-membrane proteins like MK 0646, AMG 479, and CS 1008; small-molecule inhibitors of oncogenes like Masitinib, AZD 6244 and Sarilasib; and gene therapy with TNFerade are under various phases of trial. ${ }^{26}$ It is important to realize that tumor microenvironment and cancer stem cells are critical components of carcinoma pancreas and need to be attacked. Pilot studies are currently under way to study pancreatic cancer stem cells. The information gained may lead to new avenues to identify novel tumor cell markers for diagnostic purposes and to identify new cellular targets and will provide a cell population that can be used for testing new chemotherapeutic agents, biological modifiers, and immune-based therapies. ${ }^{27-29}$

\section{Conflicts of interest}

None identified.

\section{R E F E R E N C E S}

1. Jemal A, Siegel R, Ward E, et al. Cancer statistics, 2008. CA Cancer J Clin. 2008;58:71-96.

2. Shi C, Hruban RH, Klein AP. Familial pancreatic cancer. Arch Pathol Lab Med. 2009;133:365-374. 
3. Jones $\mathrm{S}$, Zhang X, Parsons DW, et al. Core signaling pathways in human pancreatic cancers revealed by global genomic analyses. Science. 2008;321:1801-1806.

4. Jones S, Hruban RH, Kamiyama M, et al. Exomic sequencing identifies PALB2 as a pancreatic cancer susceptibility gene. Science. 2009;324:217.

5. Batty GD, Kivimaki M, Morrison D, et al. Risk factors for pancreatic cancer mortality: extended follow-up of the original Whitehall Study. Cancer Epidemiol Biomark Prev. 2009;18:673-675.

6. Landi S. Genetic predisposition and environmental risk factors to pancreatic cancer: a review of the literature. Mutat Res. 2009;681:299-307.

7. Genkinger JM, Spiegelman D, Ander-son KE, et al. Alcohol intake and pancreatic cancer risk: a pooled analysis of fourteen cohort studies. Cancer Epidemiol Biomark Prev. 2009;18:765-776.

8. Wolpin BM, Chan AT, Hartge P, et al. ABO blood group and the risk of pancreatic cancer. J Natl Cancer Inst. 2009;101:424-431.

9. Hruban RH, Maitra A, Goggins M. Update on pancreatic intraepithelial neoplasia. Int J Clin Exp Pathol. 2008;1:306-316.

10. Maitra A, Hruban RH. Pancreatic cancer. Annu Rev Pathol. 2008;3:157-188.

11. Karmazanovsky G, Fedorov V, Kubyshkin V, Kotchatkov A. Pancreatic head cancer: accuracy of CT in determination of respectability. Abdom Imaging. 2005;30:488-500.

12. Dumonceau JM, Vonlaufen A. Pancreatic endoscopic retrograde cholangiopanceaticography (ERCP). Endoscopy. 2007;39:124-130.

13. Mayo SC, Austin DF, Sheppard BC, Mori M, Shipley DK, Billingsley KG. Evolving preoperative evaluation of patients with pancreatic cancer: does laparoscopy have a role in the current era? J Am Coll Surg. 2009;208:87-95.

14. Harsha HC, Kandasamy K, Ranganathan P, et al. A compendium of potential biomarkers of pancreatic cancer. PLoS Med. 2009;6(4): e1000046.

15. Berger AC, Garcia Jr M, Hoffman JP, et al. Postresection CA 199 predicts overall survival in patients with pancreatic cancer treated with adjuvant chemoradiation: a prospective validation by RTOG 9704. J Clin Oncol. 2008;26:5918-5922.

16. Hess V, Glimelius B, Grawe P, et al. CA 19-9 tumour-maker response to chemotherapy in patients with advanced pancreatic cancer enrolled in a randomized controlled trial. Lancet Oncol. 2008;9:132-138.
17. Greenhalf W, Grocock C, Harcus M, Neoptolemos J. Screening of high-risk families for pancreatic cancer. Pancreatology. 2009;9:215-222.

18. Edge SB, Byrd DR, Compton CC, Fritz AG, Greene FL, Trotti A, eds. AJCC Cancer Staging Manual. 7th ed. New York: Springer; 2011.

19. Pawlik TM, Laheru D, Hruban RH, et al. Evaluating the impact of a-single-day multidisciplinary clinic on the management of pancreatic cancer. Ann Surg Oncol. 2008;15:2081-2088.

20. Katz MH, Wang H, Fleming JB, et al. Long-term survival after multidisciplinary management of respected pancreatic adenocarcinoma. Ann Surg Oncol. 2009;16:836-847.

21. Tseng JF, Tamm EP, Lee JE, Pisters PW, Ewans DB. Venous resection in pancreatic cancer surgery. Best Pract Res Clin Gastroenterol. 2006:20349-20364.

22. Hernandez JM, Cowgill SM, Al-Saadi S, et al. CA 19-9 velocity predicts disease-free survival and overall survival after pancreatectomy of curative intent. J Gastrointest Surg. 2009;13:349-353.

23. Slidell MB, Chang DC, Cameron JL, et al. Impact of total lymph node count and lymph node ratio on staging and survival after pancreatectomy for pancreatic adenocarcinoma: a large, population-based analysis. Ann Surg Oncol. 2008;15:165-174.

24. Pausawasdi N, Scheiman J. Endoscopic evaluation and palliation for pancreatic adenocarcinoma: current and future operations. Curr Opin Gastroenterol. 2007;23:515-521.

25. Herman JM, Swartz MJ, Hsu CC, et al. Analysis of fluorouracilbased adjuvant chemotherapy and radiation after pancreaticoduodenectomy for ductal adenocarcinoma of the pancreas: results of a large, prospectively collected database at the Johns Hopkins Hospital. J Clin Oncol. 2008;26:3503-3510.

26. Murugesan SR, King CR, Osborn R, et al. Combination of human tumor necrosis factor-alpha (hTNF-alpha) gene delivery with gemcitabine is effective in models of pancreatic cancer. Cancer Gene Ther. 2009;16:841-847.

27. Faca VM, Song KS, Wang H, et al. A mouse to human search for plasma proteome changes associated with pancreatic tumor development. PLoS Med. 2008;5(6): e123.

28. Rajesh Kumar NV, Tan AC, De Oliveira E, et al. Antitumour effects and biomarkers of activity of AZD0530, a Src inhibitor, in pancreatic cancer. Clin Cancer Res. 2009;15:4138-4146.

29. Hewish M, Chau I, Cunningham D. Insulin-like growth factor 1 receptor targeted therapeutics: novel compounds and novel treatment strategies for cancer medicine. Recent Pat Anticancer Drug Discov. 2009;4:54-72. 